\title{
A Novel Approach to Cattle Health Monitoring for Maximizing Dairy Output Using LoRa IoT Technology
}

\author{
Adithya Sampath ${ }^{1}$, P Meena ${ }^{2}$ \\ Student, Electrical \& Electronics Engineering, BMS College of Engineering, Bengaluru, India ${ }^{1}$ \\ Head of Department, Electrical \& Electronics Engineering, BMS College of Engineering, Bengaluru, India ${ }^{2}$
}

\begin{abstract}
The past few decades have seen rapid growth in consumption of dairy products in several parts of the developing world. According to a study by Food and Agriculture Organization under the United Nations, it is predicted that there will be $68 \%$ higher demand for milk and milk products by 2050 . With the ever growing demand a major concern is the low milk yield of cows. The potential of increase in dairy consumption remains significant, as population levels continue to grow in developing countries, especially in countries like India. The focus of this work is to design a lightweight and smart IoT wearable device strapped around the cow's neck to help farmers to monitor the vitals of cows through various sensors and hence monitor cattle health, which contributes to a healthy reproductive cycle and therefore leads to an improved milk yield. The IoT wearable device measures temperature, position activity and behaviour (Eating, sleeping, lameness). LoRa communication protocol is the IoT technology used for the communication of this information. The IoT wearable is the LoRa device which transmit the cattle's health information and the data is received by a LoRa gateway, which is the data hub of all received sensor data from the cattle. The collected data is then sent to cloud from the LoRa gateway. Data analytics is done on the cloud and a report is generated detailing the health of each cow in the farm. Based on this report the farmer can approach a vet, nutritionist, agronomist to get actionable intelligence to improve the cow's milk yield. The results obtained from the testing of the PoC prototype on cattle in a farm are presented.
\end{abstract}

Keywords: Improve Dairy Output, IoT Wearable Device, LoRa Protocol, Cloud Data Analytics

\section{INTRODUCTION}

The importance of the dairy industry in a country like India needs no emphasizing. India has one of the largest resources of livestock, which plays a very important role in the national economy and in the socio-economic development of millions of rural households in the country. India has one of the largest stocks of cattle and buffaloes more than 50 percent of the world's buffaloes and 20 percent of its cattle, but as observed in Fig 1, the per cow milk production is one of the lowest in the world. [1] This is the major concern for most dairy farmers in the country. Farmers use state of the art technology to help increase food production for a growing world. Through the use of technology, each farmer is now able to feed 155 people today, compared to 1940, when one farmer could feed only 19 people. Improvements in technology has increased the efficiency in the farming process number and requires lesser people to work on the farm. This however, isn't enough due to the ever-increasing demands worldwide as there is a concern about the dairy output. The Figures. 1 and 2 are indicative of this.

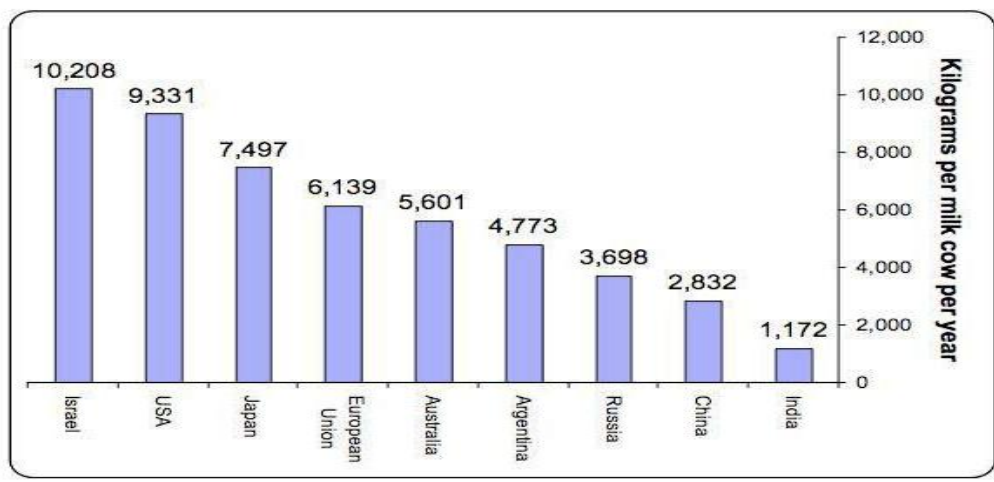

Fig. 1: Kg yield of cows per year in different countries. [Source: Dairy school-Israeli dairy industry] 
Vol. 8, Issue 6, June 2019
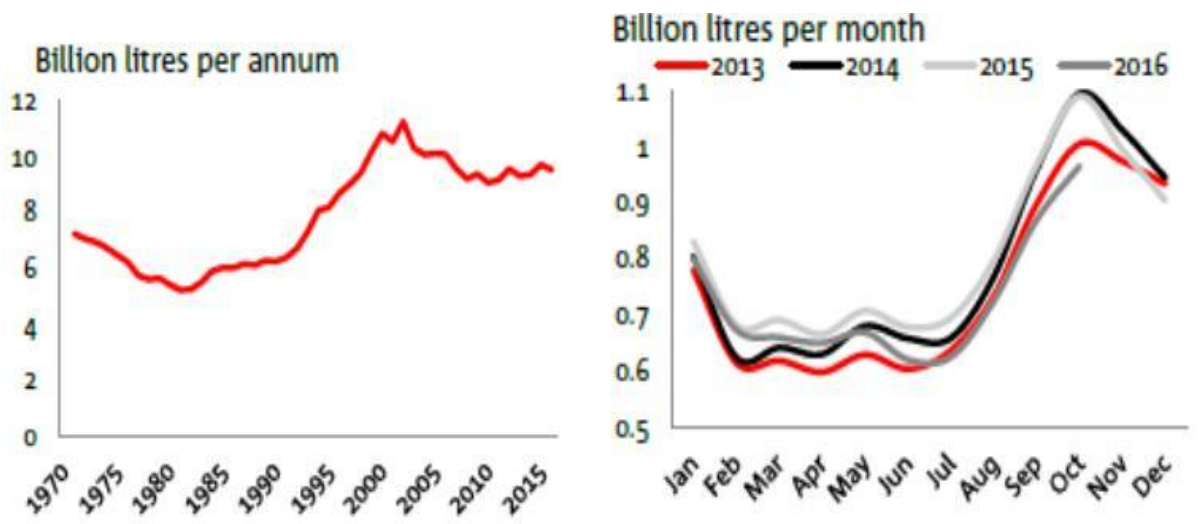

Fig. 2: Annual vs Monthly Milk production in Australia. milk production plunged last year by $11.4 \%$. [Source: Dairy Australia, ABARES]

To address this concern, a technology-based solution is proposed to increase the milk yield per cow. This can be done by designing a lightweight IoT device that goes around the cow's neck to monitor the vitals of every cow in the farm and applying data analytics to the collected data and give a detailed report to the farmer, who can then approach veterinarian, agronomist or a nutritionist with actionable intelligence and take informed decisions to maximizing the yield of every cow in the farm. The paper covers the vitals measured and their significance in Section (II), the design of the wearable and gateway with their functional methodology of the end-to-end setup in Section in Section (III), the results of testing the device in a live farm in Section (IV), Conclusion in Section (V) and References in Section (VI)

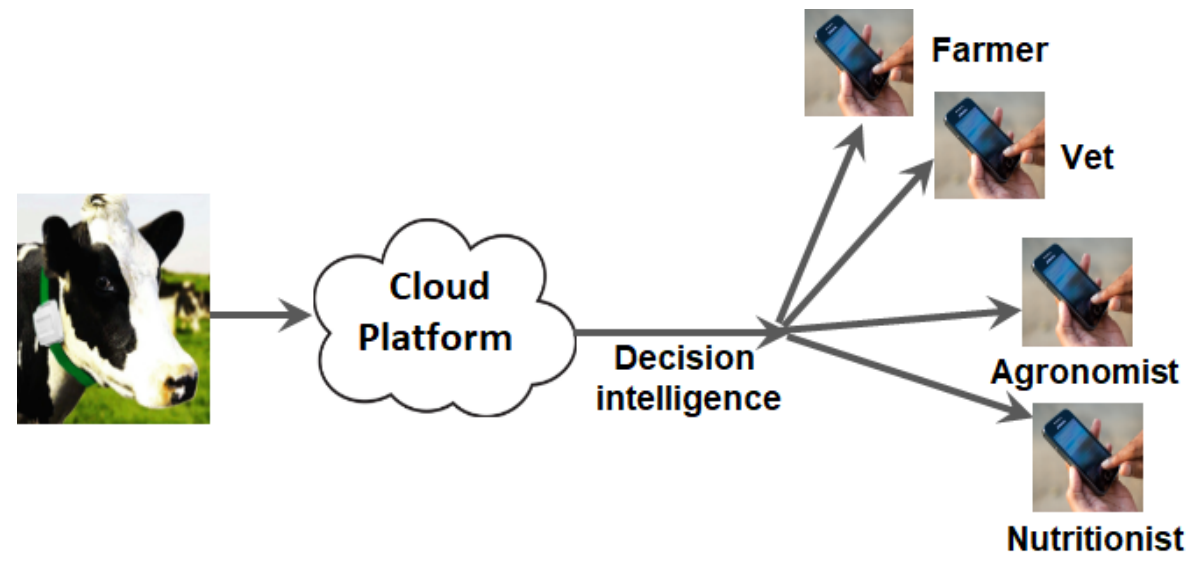

Fig. 3: High-level representation of proposed solution

\section{SIGNIFICANCE OF SENSOR MEASUREMENTS}

A. Temperature:

Measuring body temperature is an important aspect as it is part of the definition of puerperal metritis [3].

All antibiotic treatment decisions are based on body temperature. Several factors can influence the measurement of the body temperature (type of thermometer, insertion depth, skills of the investigator) as well as the cow's body temperature (days in milk, parity, time of the day, climate at calving). [3] The normal core body temperature of a healthy, resting cow is stated on average to be 101.5 degrees Fahrenheit (38.6 degree Celsius), where a fever is diagnosed when the temperature > 103.0 F. [4]

\section{B. Position Activity:}

Global positioning System (GPS) tracking of farm animals has several benefits that can help a ranch or dairy farm thrive. When you use GPS tracking on your farm, one can:

1. Finding missing livestock that has wandered away from the herd

2. Determine how much livestock you can commit to individual fields

3. Rotate fields to prevent erosion and pollution

4. Keep track of short-term and long-term herd behaviours that will help manage animals 
Vol. 8, Issue 6, June 2019

C. Behaviour:

There are behaviour changes when animals fall sick which can be linked to specific illnesses and hence behaviour changes. For example, cattle with acute lameness spend less time at the feed and eat lesser. One can also detect changes in behaviour for some illnesses before there are any clinical signs. Wearable technology for livestock are useful as early warning systems to farmers, which use behavioural changes to detect disease development at an early stage. The risk of diseases has a large effect on the economics of a farm, through payment for treatments and loss of production from the infected animals, as well as on animal welfare. Sick animals will normally be more lethargic, isolate themselves and lose their appetite. This in turn affects the reproductive cycle and hence the dairy output of the cow. [5]

\section{DESIGN OF WEARABLE AND GATEWAY}

\section{A. Why Lora:}

Unlike similar work [6][7][8] which use a WiFi network to transmit sensor data to a server, LoRa (short for long range) IoT technology is used here. There are a multitude of benefits to using LoRa over any other communication technology:

1) LoRa protocols put least burden on device battery. Provides longest battery lifetime on IoT devices.

2) Provides Line-of-Sight range of up to $5 \mathrm{~km}$ in rural setup.

3) Low cost (RF-LORA-868 module cost roughly Rs1500 in India)

4) Suitable for both indoor and outdoor applications; Like Wi-Fi, LoRa operates in the unlicensed band and supports indoor applications; like cellular, LoRa Technology is highly secure from end devices to the application server, and is suitable for outdoor application

\begin{tabular}{|c|c|c|c|c|c|}
\hline Feature & LoRaWAN & Narrow-Band & $\begin{array}{l}\text { LTE } \\
\text { Cat-1 }\end{array}$ & LTE Cat-M & NB-LTE \\
\hline Modulation & SS Chirp & UNB/GSK/BPSK & OFDMA & OFDMA & OFDMA \\
\hline Rx bandwidth & $500-125 \mathrm{kHz}$ & $100 \mathrm{~Hz}$ & $20 \mathrm{MHz}$ & $\begin{array}{l}20-1.4 \\
\mathrm{MHz}\end{array}$ & $\begin{array}{l}200 \\
\mathrm{KHz}\end{array}$ \\
\hline Data Rate & $\begin{array}{l}290 \text { bps }-50 \\
\text { Kbps }\end{array}$ & $\begin{array}{l}100 \mathrm{bit} / \mathrm{sec} \\
12 / 8 \text { bytes max }\end{array}$ & $\begin{array}{l}10 \\
\text { Mbps }\end{array}$ & $\begin{array}{l}200 \mathrm{kbps}- \\
1 \mathrm{Mbps}\end{array}$ & $\begin{array}{l}20 \\
\text { Kbps }\end{array}$ \\
\hline $\begin{array}{l}\text { Battery lifetime } \\
\text { - } 2000 \text { mAh }\end{array}$ & $\begin{array}{l}105 \text { months } \\
\text { ( } \sim 9 \text { years) }\end{array}$ & $\begin{array}{l}90 \text { months ( } 7.5 \\
\text { years) }\end{array}$ & & $\begin{array}{l}18 \text { months } \\
\text { (1.5 years) }\end{array}$ & \\
\hline Security & Yes & No & Yes & Yes & Yes \\
\hline
\end{tabular}

Fig. 4: Advantages of LoRaWAN over other communication protocols [9]

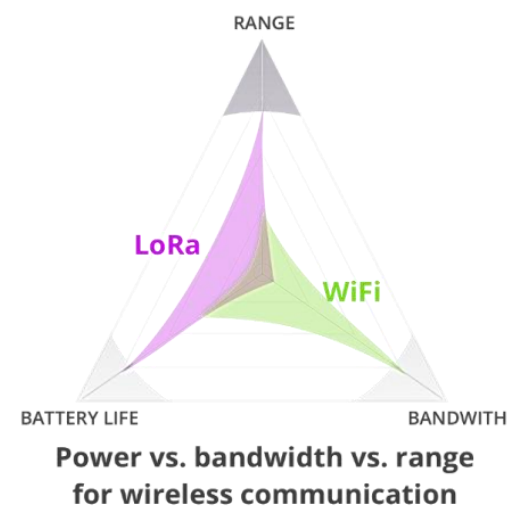

Fig5: LoRa vs Wifi comparison [10]

Wifi isn't practically feasible on IoT devices due to its power consumption and low range. Indian farms span for Kilometres and LoRa having a LoS range of up to $5 \mathrm{~km}$ (in open rural farms lands without obstructions) is ideal. Another noteworthy feature is its extremely low power consumption (20 mA in "idle" mode and topped out at less than 
Vol. 8, Issue 6, June 2019

$110 \mathrm{~mA}$ during transmission) hence providing a battery lifetime of an average 6 months; this ensures farmers don't have to change batteries on the IoT devices regularly. Keeping the above factors in mind, LoRa was chosen.

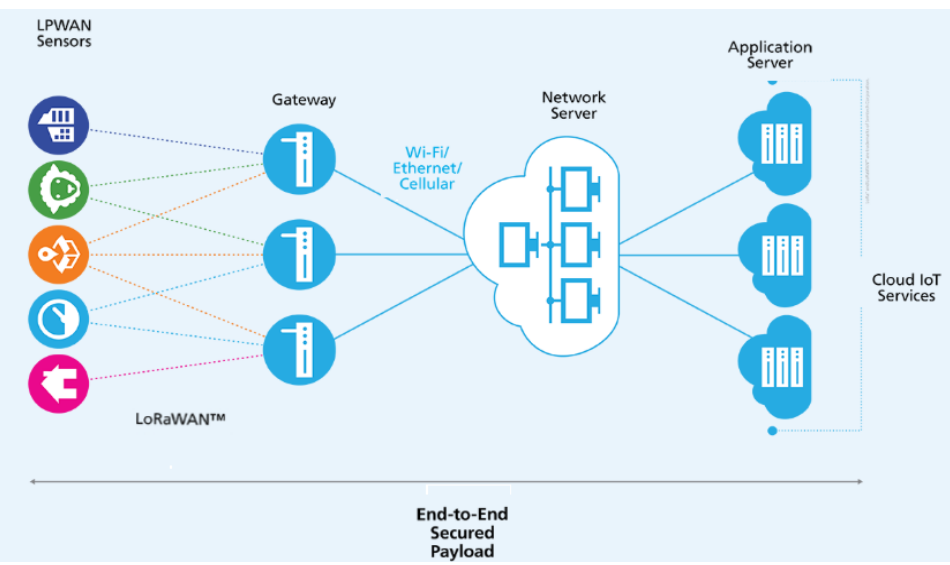

Fig. 6: LoRaWAN network (Source: https://www.semtech.com/lora/what-is-lora)

B. LoRa IOT Wearable

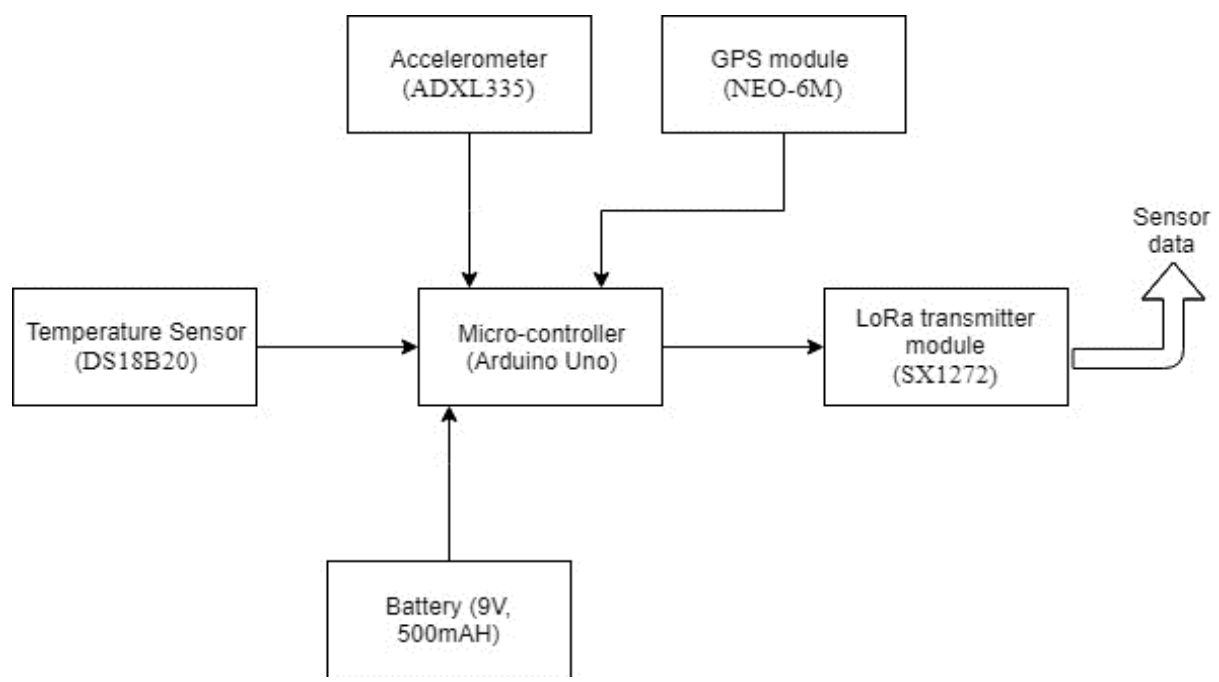

Fig. 7: Block diagram of LoRa IoT wearable

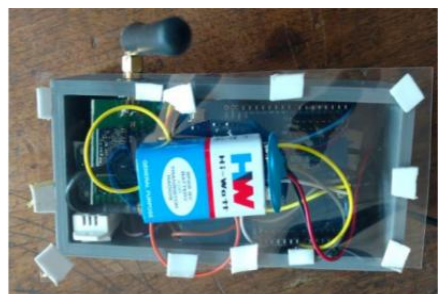

Fig. 8: 3D printed LoRa IoT wearable device

Table I: Components of LoRa IoT Wearable

\begin{tabular}{|l|l|l|l|}
\hline $\begin{array}{c}\text { S1 } \\
\text { no. }\end{array}$ & $\begin{array}{c}\text { Senor / Component } \\
\text { name }\end{array}$ & $\begin{array}{c}\text { Senor / Component } \\
\text { type }\end{array}$ & \multicolumn{1}{|c|}{ Properties } \\
\hline 1 & Arduino Uno & Microcontroller & $\begin{array}{l}\text { Brain of the setup with Atmega 328P IC. Reads sensor data } \\
\text { from its Analog and Digital Pins, and transmits data through } \\
\text { LoRa module interface. }\end{array}$ \\
\hline 2 & SX1272 & LoRa transmitter & $\begin{array}{l}\text { Is Semtech's LoRa long range modem/transceiver which } \\
\text { transmits data in the unlicensed LoRa frequency of 868MHz. Its } \\
\text { has 6 pins and communicated with Arduino on SPI. }\end{array}$ \\
\hline 3 & DS18B20 & Temperature sensor & $\begin{array}{l}\text { The DS18B20 is a digital thermometer with 1-Wire bus that } \\
\text { requires only one digital data line (and ground) for }\end{array}$ \\
\hline
\end{tabular}


Vol. 8, Issue 6, June 2019

\begin{tabular}{|l|l|l|l|}
\hline & & & $\begin{array}{l}\text { communication with Arduino Uno. Measures Temperatures } \\
\text { from }-55^{\circ} \mathrm{C} \text { to }+125^{\circ} \mathrm{C}\left(-67^{\circ} \mathrm{F} \text { to }+257^{\circ} \mathrm{F}\right) \text {. Highly accurate with } \\
\pm 0.5^{\circ} \mathrm{C} \text { Accuracy from }-10^{\circ} \mathrm{C} \text { to }+85^{\circ} \mathrm{C} .\end{array}$ \\
\hline 4 & ADXL335 & Accelerometer & $\begin{array}{l}\text { The ADXL335 is a 3-axis sensing low power accelerometer } \\
\text { with } \pm 3 \text { g measurement range. Its connected to the analog pins } \\
\text { of Arduino Uno. }\end{array}$ \\
\hline 5 & UBLOX NEO-6M & GPS Module & $\begin{array}{l}\text { NEO-6M is cost effective, high-performance GPS receiver with } \\
\text { a built-in } 25 \times 25 \mathrm{x} 4 \mathrm{~mm} \text { ceramic antenna, which provides a } \\
\text { strong satellite search capability. It has an EEPROM to store } \\
\text { data during power failure. }\end{array}$ \\
\hline 6 & $9 \mathrm{~V}$ battery & Power supply & $9 \mathrm{~V}, 500 \mathrm{mAH}$ battery. \\
\hline
\end{tabular}

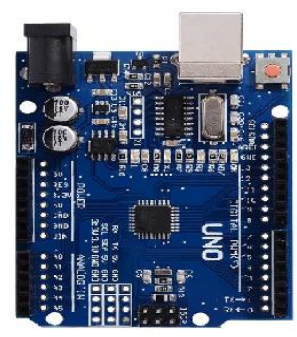

(i)

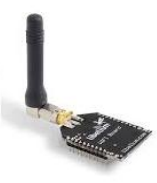

(ii)

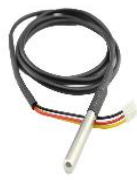

(iii)

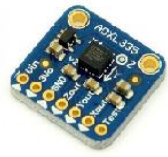

(iv)

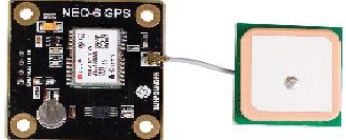

(v)

Fig. 9: List of components encased in the 3D printed wearable (left to right: Arduino Uno, SX1272 LoRa Transceiver, DB18B20 Temperature sensor, ADXL335 Accelerometer, Ublox Neo-6m GPS module)

The LoRa IoT wearable device is a $15 \mathrm{~cm}$ x $7 \mathrm{~cm}$ x 7cm 3D printed case which encloses the Microcontroller, the sensors (Temperature sensor, Accelerometer, GPS module), 9V battery and the Semtech SX1272 LoRa transceiver. Slot opens in the 3D printed case are made to give space for the antenna of LoRa transceiver (projected outward, away from the cow) and the DB18B20 temperature sensor (projected inward, in contact with the cow's neck). The Arduino Uno is placed at the base of the case. On it a mini-breadboard is placed, for a common ground and shorting power supply for Arduino UNO and sensors. The antenna of the Neo-6M GPS module is placed on top of the breadboard to measure GPS information accurately. The ADXL335 accelerometer is placed next to the Arduino Uno on the bottom of the case. The Arduino Uno is programmed to transmit sensor data only every 15 minutes; rest of the time the LoRa module is in "idle state" and consumes just 10mA. Based on our tests in the college campus, the LoRa transceiver sends data with fidelity up to $500-750 \mathrm{~m}$, due to presence of various obstacles and obstructions. Even upon regular continuous tests over a period of 1 month, the battery was never replaced. No internet connection is required to transmit data here.

C.

LoRa IoT Gateway

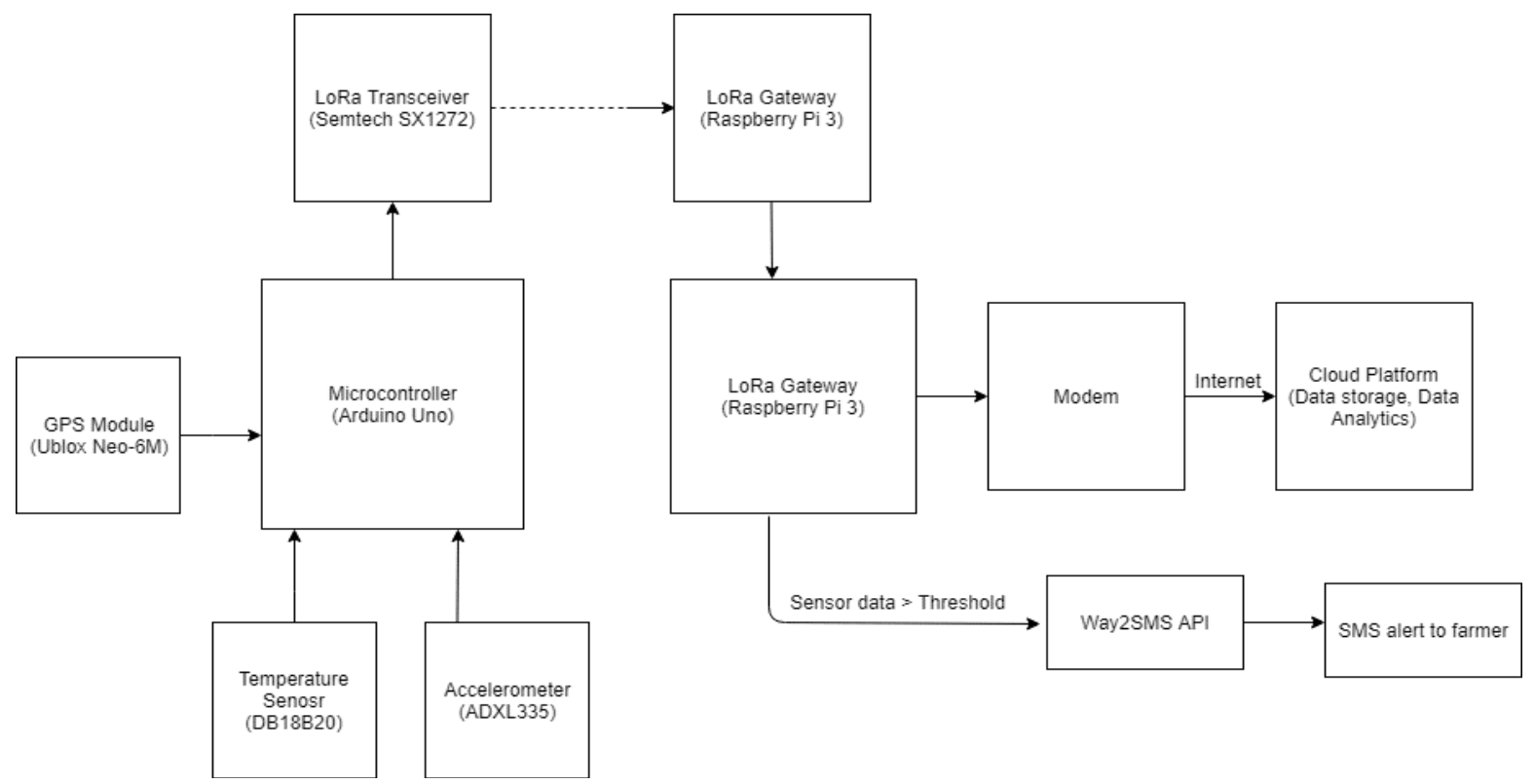

Fig. 10: End-to-End Block diagram of LoRa IoT setup. 


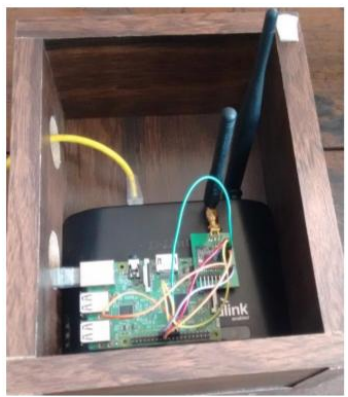

Fig. 11: LoRa Gateway Setup

The LoRa gateway is a Raspberry Pi 3, with a Semtech 1272 LoRa Transceiver to receive sensor data from all LoRa IoT wearables in range. The LoRa gateway is set up on Raspbery Pi 3 using [11]. An internet connection is required for sending collected sensor data to the cloud, so we compile and configure the gateway to set the gateway ID and WiFi access point SSID. Once set up, the Raspberry Pi gateway is ready to receive sensor data. The sensor data is collected, post-processed at the gateway and logging begins on the default ThingSpeak channel to store and visualize data of each device on the cloud. During post-processing, some thresholds of sensor values are set, to alert farmer in case of sudden anomaly in a cow's health. Way2SMS API is configured to send SMS to farmers in case collected sensor data cross the threshold.

D. ThingSpeak Cloud Platform

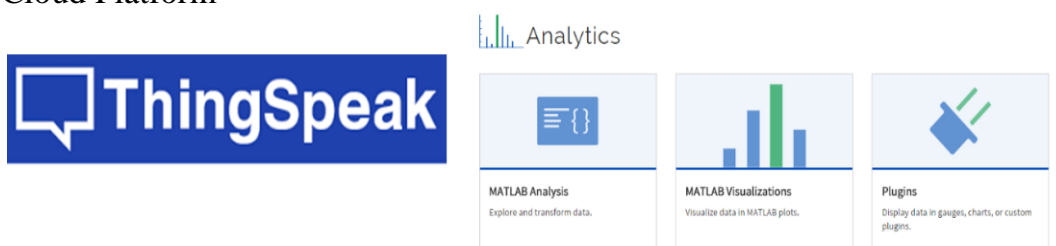

Fig. 12: ThingSpeak platform (Source: https://thingspeak.com/)

ThingSpeak is a simple and very popular IoT cloud. Once a Channel is created on the platform, any user can stream data to this channel from a registered gateway. Once a Channel is created, the Channel ID, ReadAPI and WriteAPI keys of the channel must be added in the config file of the LoRa gateway. On the gateway end, the user must configure the post-processing script to send collected and processed data to the ThingSpeak channel created. After we build this configuration, the senor data received from LoRa IoT devices will be post-processed and uploaded, stored and visualized on the ThingSpeak channel. Here MATLAB Analytics can be done on the collected data to draw inferences on the health of each cow. Here Generalized Linear Regression is implemented on MATLAB to predict the most probable temperature of the cow at a given time. Also implemented a MAX counter to identify and store the maximum temperature of the cow for the past hour.

\section{RESULTS}

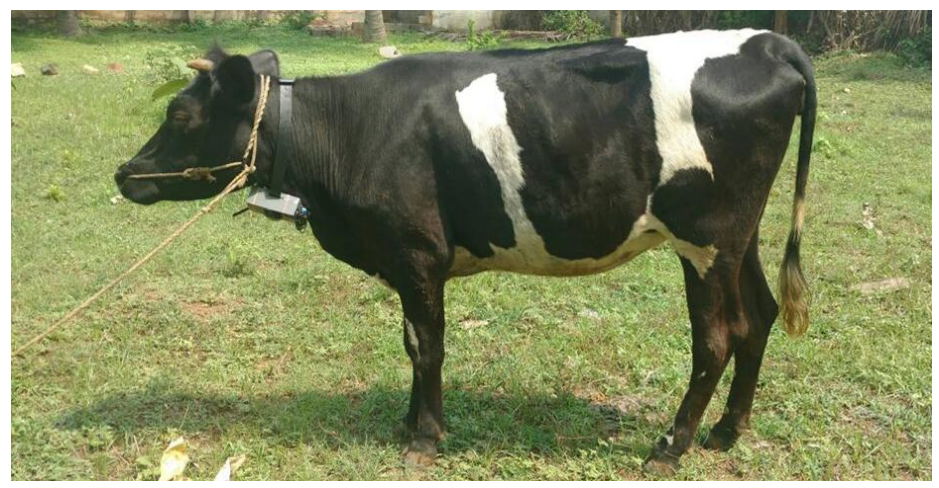

Fig. 13: The LoRa IoT wearable device tested on a cow

The wearable designed and developed was tested for its intended functionality on a cow in a farm. The testing was done for different activity conditions such as while the cow was at rest, on the move. The result shown below is a plot of accelerometer in all three reference directions with respect to time. 


\section{International Journal of Advanced Research in Computer and Communication Engineering}

Vol. 8, Issue 6, June 2019

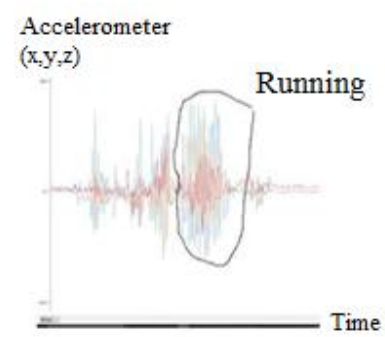

(i)

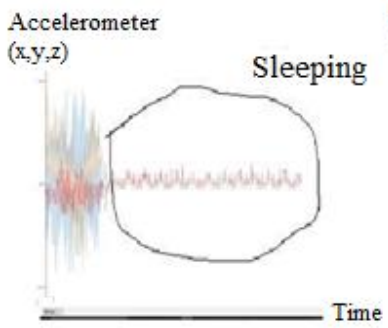

(ii)

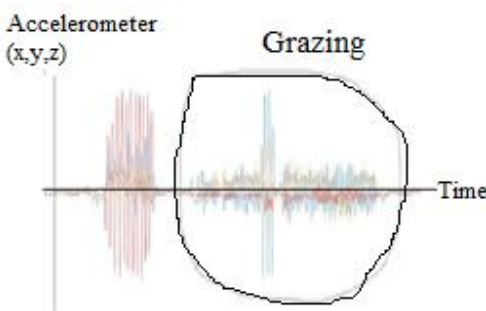

(iii)

Fig. 14: Plot of Accelerometer (x, y, z) vs Time (left to right: Activity chart for running, sleeping/idle, grazing)
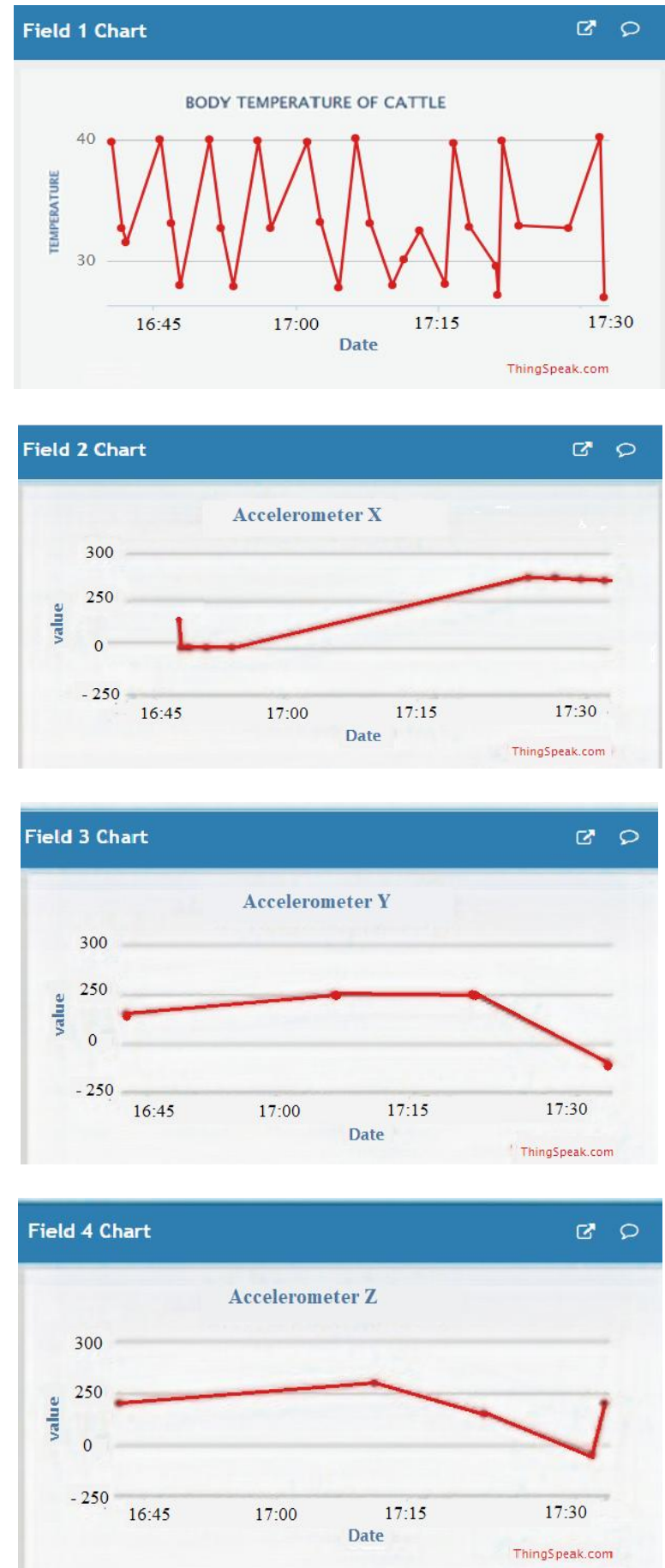

Figure.15: The various sensor data received (Temperature, Accelerometer X,Accelerometer Y,Accelerometer Z) and visualized on the ThingSpeak cloud platform. Here the data was plotted in real-time with time stamping. 
Vol. 8, Issue 6, June 2019

QThingSpeak" channels - Apps Community Support - Howto Buy Account - Sien Out

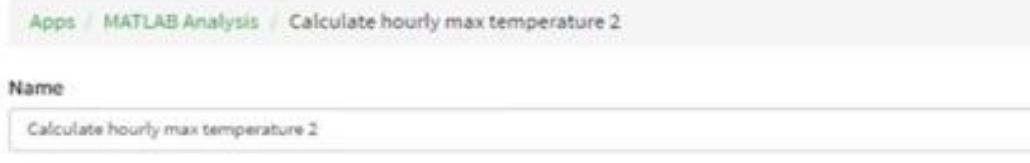

MATLAB Code

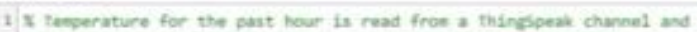

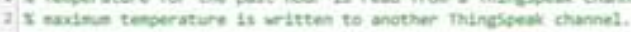

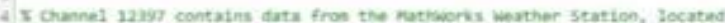

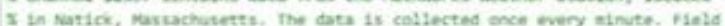
I a contafins temperature tata.

I channel to to read data frot

reaschanne1ID - 22074s:

S Tempersture 1 ield tid

Temperaturef lelote $=1$ :

5 Chunnel had Nit bey

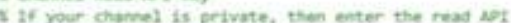

s rey petien the '." pele

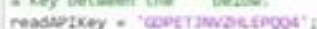

\section{Save andiun}

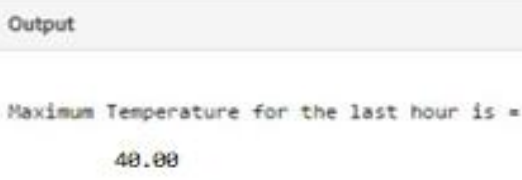

Output

Maxinum Tenperature for the last hour is a

49.90

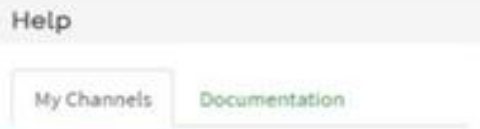

\section{Now Charnel}

Chansel info

Name: LoRa Gatewa

Channel 10: 210745

Accens Public

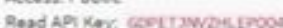

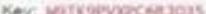

Fivids:

i: Temperature

2: aces

zacey

4: ace:

5. Lat

6.len

vame: Average Temperature

Channel 10: 273478

Accents Public

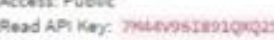

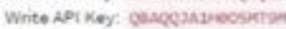

Fieldr:

1. Ave Temp

Figure.16: MATLAB analytics on the sensor data received on the ThingSpeak cloud platform.

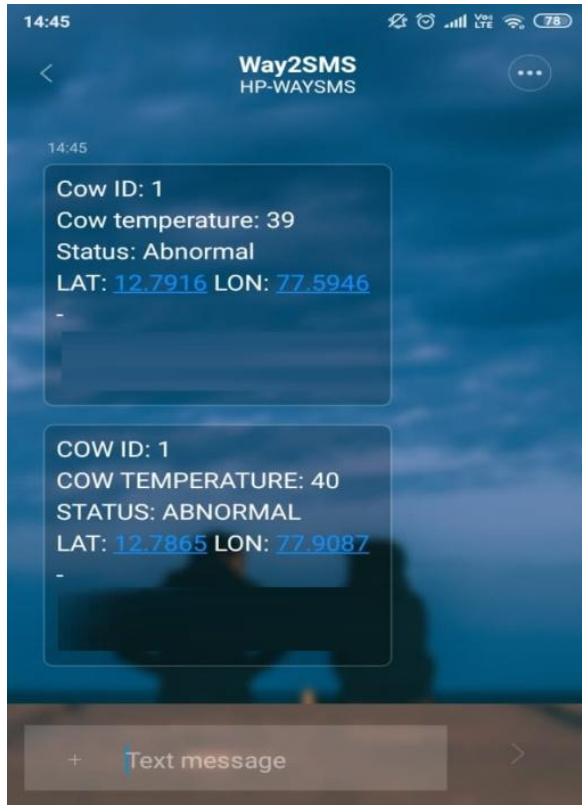

Figure.17: SMS alert received from Gateway through SMS API when sensor value crosses threshold

\section{CONCLUSION}

The LoRa IoT wearable device on each cow on the farm monitors the temperature, activity and position of the cow and sends it to a LoRa gateway using LoRa protocol. The Cloud Analytics done on the sensor data received at the gateway is used to generate a report of actionable information for the farmer and the veterinarian to act on. The outcomes of this are: 1) Early identification of diseases 2) Activity and GPS tracking of cattle 3) Identify period of healthy reproduction cycle hence 4) Increased milk output. 
Vol. 8, Issue 6, June 2019

\section{ACKNOWLEDGEMENT}

The authors deeply acknowledge the support received from Technical Education Quality Improvement Program PhaseII [ A World Bank funding initiative] for the financial support extended to build the working prototype. The authors acknowledge Dr Vijay Viswanathan, CEO \& Co-Founder, IoTAg, Dr Matthew Petersen, CTO \& Co-Founder, IoTAg, for the opportunity provided to design a prototype for their start-up.

\section{REFERENCES}

[1]. Henning Steinfeld, "Livestock, Environment and Development Initiative (LEAD)", Animal Production and Health Division, FAO

[2]. LeBlanc SJ et al (2011) Reproductive tract defense and disease in postpartum dairy cows. Theriogenology 76(9):1610.

[3]. Fuquay JW, Chapin LT, Brown WH (1980) Short term post-partum heat stress in dairy cows. Int J Biometeorol 24:141-148

[4]. Herbut P, Angrecka S, Walczak J. Environmental parameters to assessing of heat stress in dairy cattle-a review. Int J Biometeorol. 2018;62(12):2089-2097. doi:10.1007/s00484-018-1629-9

[5]. Cate Williams IBERS, Aberystwyth University. (2019) "Metabolic Profiling of dairy cows to improve efficiency and production". [Online]. Available: http://www.businesswales.gov.wales, January 2019

[6]. Leena Narayan, Dr. T. Muthumanickam and Dr. A. Nagappan, “Animal Health Monitoring System using Raspberry Pi and Wireless Sensor", International Journal of Scientific Research and Education (IJSRE), Volume 3 Issue 5, May 2015

[7]. Anselemi B.Lukonge, Dr. Shubi Kaijage and Ramadhani S. Sinde, "Review of Cattle Monitoring System using Wireless Network" , International Journal of Engineering and Computer Science (IJECS), Volume 3, Issue 5, May 2014

[8]. L. Nagl, R. Schmitz, S. Warren, T. S. Hildreth, H. Erickson and D. Andresen, "Wearable Sensor System for Wireless State-of-Health Determing in Cattle"

[9]. 3GLTEInfo Article, "LoRa Vs LTE: LoRa Advantages Over Cellular and Local Area Networks", [Online]. Available: http://www.3glteinfo.com/lora/lora-advantages/

[10]. Samuel L Martinet, “3 Differences between LoRa and WiFi”, [Online]. Available: https://medium.com/@samLmartinet/lora-vs-wifi-3questions-d9c93137fca

[11]. C. Pham, LIUPPA laboratory, University of Pau, France. “A DIY low-cost LoRa gateway” [Online]. Available: http://web.univ-pau.fr/ cpham 